\title{
Idempotent Method for Dynamic Games and Complexity Reduction in Min-Max Expansions
}

\author{
William M. McEneaney
}

\begin{abstract}
In recent years, idempotent methods (specifically, max-plus methods) have been developed for solution of nonlinear control problems. It was thought that idempotent linearity of the associated semigroup was required for application of these techniques. It is now known that application of the max-plus distributive property allows one to apply the maxplus curse-of-dimensionality-free approach to stochastic control problems. Here, we see that a similar, albeit more abstract, approach can be applied to deterministic game problems. The main difficulty is a curse-of-complexity growth in the computational cost. Attenuation of this effect requires finding reduced-complexity approximations to min-max sums of maxplus affine functions. We demonstrate that that problem can be reduced to a pruning problem.
\end{abstract}

\section{INTRODUCTION}

In recent years, idempotent methods have been developed for solution of nonlinear control problems. (Note that idempotent algebras are those for which $a \oplus a=a$ for all $a$; this class includes the well-known max-plus algebra.) Most notably, max-plus methods have been applied to deterministic optimal control problems. These consist of max-plus basis methods, exploiting the max-plus linearity of the associated semigroup [1], [2], [8], [11], [15], [18] [19], and max-plus curse-of-dimensionality-free methods which exploit the maxplus additivity and the invariance of the set of quadratic forms under the semigroup operator [11], [14], [16]. These methods achieved truly exceptional computational speeds on some classes of problems. Recently, use of the idempotent distributive property has allowed some first expansions of max-plus curse-of-dimensionality-free concepts into the domain of stochastic control problems [13], [22], [23].

In this paper, we use some similar, but more abstract, tools which will bring game problems into the realm under which curse-of-dimensionality-free idempotent methods will be applicable. We will first demonstrate how one may apply the min-max distributive property to develop curseof-dimensionality-free methods for discrete-time, deterministic dynamic games. We will only outline the steps in that development. The difficulty with idempotent curse-ofdimensionality-free methods for stochastic and game problems is an extreme curse-of-complexity difficulty. A means for attenuating that difficulty will be the focus of this paper. We will see that the proper space to work in is the space of max-plus convex functions. Our definition of max-plus

Research partially supported by NSF grant DMS-0307229 and AFOSR grant FA9550-06-1-0238.

Dept. of Mechanical and Aerospace Engineering, University of California San Diego, San Diego, CA 92093-0411, USA. wmceneaney@ucsd.edu convex functions differs from that in [6], [25] via a reversal of the ordering on the range space. This change creates a more useful space for our purposes here. We will note that the space of max-plus convex functions is a minmax vector space (or moduloid). This is an analogy of the useful property that the space of standard-sense convex functions is a max-plus vector space (or moduloid). We will then consider max-plus convex functions given as finite min-max sums of max-plus affine functions. Reducing the complexity of such representations is the key to practicality in an idempotent algorithm for game problems. We will demonstrate that such a complexity reduction is reduced to a pruning operation. That is, given a min-max sum of $M$ maxplus affine functions, the best approximation (in a sense to be defined) in the class of min-max sums of $N$ max-plus affine functions (with $N<M$ ) consists of a subset of the original set. This greatly reduces the solution space of the complexity reduction problem.

Due to space limitations, almost all results will be presented without their proofs. Many of these are lemmas where the proofs would be relatively short and clear. The proofs of Theorems 3.6, 3.13, 3.14 and 4.2 are included to give a sense of the analysis.

\section{IdEMPotent Method fOR Games}

We briefly describe the idempotent approach to dynamic games. The purpose here is only to outline the algorithm; this will motivate the complexity reduction problem. We will keep all control spaces finite so as to simplify the analysis.

We suppose the dynamics are governed by

$$
\begin{aligned}
& \xi_{t+1}=h\left(\xi_{t}, u_{t}, w_{t}\right), \\
& \xi_{s}=x \in G \subseteq \mathbb{R}^{I},
\end{aligned}
$$

where $s$ is the initial time. We suppose $u_{t} \in \mathcal{U}$ and $w_{t} \in$ $\mathcal{W}$ for all $t$, with $W \doteq \# \mathcal{W}$ (the cardinality of $\mathcal{W}$ ) and $U=\# \mathcal{U}$. We assume $h((\cdot, u, w)$ maps $G$ into $G$ for all $u \in \mathcal{U}, w \in \mathcal{W}$. Time is discrete with $t \in] s, T[\doteq\{s, s+$ $1, s+2, \ldots T\}$, and this notation will be use throughout. Also for simplicity, we assume only a terminal cost, which will be $\phi: G \rightarrow \mathbb{R}$. We let $\mathcal{U}$ be the minimizing player's control set, and $\mathcal{W}$ be the maximizing player's control set. The payoff, starting from any $(t, x) \in] s, T[\times G$ will be

$$
J_{t}\left(x, u_{] t, T-1}\left[, w_{] t, T-1[}\right)=\phi\left(\xi_{T}\right),\right.
$$

where $u_{t t, T-1[}$ denotes a sequence of controls, $\left\{u_{t}, u_{t+1}, \ldots u_{T-1}\right\}$, with similar meaning for $w_{t, T-1[}$. We will work with the upper value, although the analysis for 
the lower value is analogous. The upper value for any time $t \in] s, T-1[$ is

$$
V_{t}(x)=\max _{\tilde{w}^{t} \in \widetilde{W}^{t}} \min _{u_{] t, T-1[} \in \mathcal{U}^{T-t}} J_{t}\left(x, u_{] t, T-1[} \cdot \tilde{w}\left(u_{] t, T-1[}\right)\right.
$$

where $\widetilde{W^{t}}=\left\{\tilde{w}^{t}: \mathcal{U}^{T-t} \rightarrow \mathcal{W}^{T-t}\right\}$. The associated dynamic programming equation (which we present without proof) is

$$
V_{t}(x)=\min _{u \in \mathcal{U}} \max _{w \in \mathcal{W}} V_{t+1}(h(x, u, w)) .
$$

Suppose $\phi$ takes the form

$$
\phi(x)=\min _{z_{T} \in \mathcal{Z}_{T}} g_{T}\left(x ; z_{T}\right)
$$

where we let $Z_{T}=\# \mathcal{Z}_{T}<\infty$. Then,

$$
V_{T}(x)=\min _{z_{T} \in \mathcal{Z}_{T}} g_{T}\left(x ; z_{T}\right) .
$$

Combining (5) and (6), one has

$$
V_{T-1}(x)=\min _{u \in \mathcal{U}} \max _{w \in \mathcal{W}} \min _{z_{T} \in \mathcal{Z}_{T}} g_{T}\left(h(x, u, w) ; z_{T}\right) .
$$

We now introduce the relevant idempotent algebras. As usual, the max-plus algebra (more properly, the max-plus commutative semifield) is defined by

$$
a \oplus b \doteq \max \{a, b\}, \quad a \otimes b \doteq a+b,
$$

operating on $\mathbb{R}^{-} \doteq \mathbb{R} \cup\{-\infty\}$. In the min-max algebra (more properly, the min-max commutative semiring), the addition and multiplication operations are defined as

$$
a \oplus^{\vee} b \doteq \min \{a, b\}, \quad a \otimes^{\vee} b \doteq \max \{a, b\},
$$

operating on $\overline{\mathbb{R}} \doteq \mathbb{R} \cup\{-\infty\} \cup\{+\infty\}$, where we note that $+\infty \oplus^{\vee} b=b$ for all $b \in \overline{\mathbb{R}}$ and $+\infty \otimes^{\vee} b=+\infty$ for all $b \in$ $\overline{\mathbb{R}}$. We suppose each $g_{T}\left(\cdot ; z_{T}\right)$ is max-plus affine. In other words, $\phi$ will be formed as the lower envelope of a finite set of max-plus affine functions. In fact, we are going to think of $\phi$ as a max-plus convex function. (We will have reason to reverse the ordering on the range space, and so our definition of max-plus convex functions will look directly analogous to the definition of standard-sense convex functions.) We may write these max-plus affine $g_{T}\left(\cdot ; z_{T}\right)$ as

$g_{T}\left(x ; z_{T}\right)=\alpha^{T, z_{T}} \odot x \oplus \beta^{T, z_{T}}=\left[\bigoplus_{i \in \mathcal{I}} \alpha_{i}^{T, z_{T}} \otimes x_{i}\right] \oplus \beta^{T, z_{T}}$

where $\mathcal{I}=] 1, I[$. We will assume that the $h(\cdot, u, w)$ are maxplus linear. Specifically, we let

$$
h(x, u, w)=A(u, w) \otimes x,
$$

where here we use $\otimes$ to emphasize that this is max-plus matrix-vector multiplication. We see that

$$
\begin{gathered}
V_{T-1}(x)=\bigoplus_{u \in \mathcal{U}}^{\vee} \bigotimes_{w \in \mathcal{W}} \vee \bigoplus_{z_{T} \in \mathcal{Z}_{T}}^{\vee}\left[\beta^{T, z_{T}} \oplus \alpha^{T, z_{T}} \odot A(u, w)\right. \\
\otimes x] .
\end{gathered}
$$

Define, for any $t \in] s+1, T\left[, \widehat{\mathcal{Z}}_{t}=\left\{\hat{z}_{t}: \mathcal{W} \rightarrow \mathcal{Z}_{t}\right\}\right.$. Applying the min-max distributive property to (8),

$$
\begin{aligned}
& V_{T-1}(x)= \bigoplus_{u \in \mathcal{U}}^{\vee} \bigoplus_{\hat{z}_{T} \in \widehat{\mathcal{Z}}_{T}}^{\vee} \bigotimes_{w \in \mathcal{W}}^{\vee}\left[\beta^{T, \hat{z}_{T}(w)}\right. \\
&= \bigoplus_{u \in \mathcal{U}}^{\vee} \bigoplus_{\hat{z}_{T} \in \widehat{\mathcal{Z}}_{T}}^{\vee} \bigoplus_{w \in \mathcal{W}}\left[\beta^{T, \hat{z}_{T}(w)} \odot A(u, w) \otimes x\right] \\
&\left.\oplus \alpha^{T, \hat{z}_{T}(w)} \odot A(u, w) \otimes x\right] .
\end{aligned}
$$

Let

$$
\begin{aligned}
& \widetilde{\alpha}_{j}^{T-1, \hat{z}_{T}}(u) \doteq \bigoplus_{w \in \mathcal{W}} \bigoplus_{i \in \mathcal{I}} \alpha_{i}^{T, \hat{z}_{T}(w)} \otimes A_{i, j}(u, w) \forall j \in \mathcal{I}, \\
& \widetilde{\beta}^{T-1, \hat{z}_{T}} \doteq \bigoplus_{w \in \mathcal{W}} \beta^{T, \hat{z}_{T}(w)} .
\end{aligned}
$$

With these definitions, (9) becomes

$$
V_{T-1}(x)=\bigoplus_{u \in \mathcal{U}}^{\vee} \bigoplus_{\hat{z}_{T} \in \widehat{\mathcal{Z}}_{T}}^{\vee}\left[\widetilde{\beta}^{T-1, \hat{z}_{T}} \oplus \widetilde{\alpha}^{T-1, \hat{z}_{T}}(u) \odot x\right] .
$$

Let $Z_{T-1}=U\left(Z_{T}\right)^{W}$, and let $\left.\mathcal{Z}_{T-1}=\right] 1, Z_{T-1}[$. Let $\Gamma_{T-1}$ be a one-to-one, onto mapping from $\mathcal{U} \times \widehat{Z}_{T}$ to $\mathcal{Z}_{T-1}$, given by $z_{T-1}=\Gamma_{T-1}\left(u, \hat{z}_{T}\right)$ for each $\left(u, \hat{z}_{T}\right) \in \mathcal{U} \times \widehat{Z}_{T}$. Then,

$$
V_{T-1}(x)=\bigoplus_{z_{T-1} \in Z_{T-1}} \vee\left[\beta^{T-1, z_{T-1}} \oplus \alpha^{T-1, z_{T-1}} \odot x\right],
$$

where

$$
\begin{aligned}
\alpha^{T-1, z_{T-1}} & \doteq \widetilde{\alpha}^{T-1, \hat{z}_{T}}(u) \\
\beta^{T-1, z_{T-1}} & \doteq \widetilde{\beta}^{T-1, \hat{z}_{T}} .
\end{aligned}
$$

Repeating this process, one easily finds the following.

Theorem 2.1: For any $t \in] s+1, T[$,

$$
V_{t-1}(x)=\bigoplus_{z_{t-1} \in Z_{t-1}}^{\vee}\left[\beta^{t-1, z_{t-1}} \oplus \alpha^{t-1, z_{t-1}} \odot x\right]
$$

where

$$
\begin{aligned}
\alpha_{j}^{t-1, z_{t-1}} & \doteq \bigoplus_{w \in \mathcal{W}} \bigoplus_{i \in \mathcal{I}} \alpha_{i}^{t, \hat{z}_{t}(w)} \otimes A_{i, j}(u, w) \quad \forall j \in \mathcal{I}, \\
\beta^{t-1, z_{t-1}} & \doteq \bigoplus_{w \in \mathcal{W}} \beta^{t, \hat{z}_{t}(w)}
\end{aligned}
$$

where $\left(u, \hat{z}_{t}\right)=\Gamma_{t-1}^{-1}\left(z_{t-1}\right)$ for all $x \in \mathbb{R}^{I}, z_{t-1} \in \mathcal{Z}_{t-1}$, and $\Gamma_{t-1}$ is a one-to-one, onto mapping from $\mathcal{U} \times \widehat{\mathcal{Z}}_{t}$ to $\left.\mathcal{Z}_{t-1} \doteq\right] 1, Z_{t-1}\left[\right.$, with $Z_{t-1}=U\left(Z_{t}\right)^{W}$.

The difficulty emerges through the iteration $Z_{t-1}=$ $U\left(Z_{t}\right)^{W}$; in a naive application of this approach, the number of max-plus affine functions defining the value would grow extremely rapidly. This implies that the second piece of the algorithm must be complexity reduction in the representation at each step. This will be the focus of the paper. 


\section{General Complexity Reduction Problem And CONTEXT}

Certain function spaces may be spanned by infima of maxplus affine functions, that is, any element of the space may be represented as an infimum of a set of max-plus affine functions. It is often useful to employ max-plus polynomial functions as well as affine. By definition, any function in such a space as the above has an expansion, $f(x)=\inf _{\lambda \in \Lambda} \psi_{\lambda}(x)$, for some index set $\Lambda$, where the $\psi_{\lambda}$ are max-plus affine. If the expansion is guaranteed to be countably infinite, we would write

$$
f(x)=\inf _{i \in \mathbf{N}} \psi_{i}(x)=\bigoplus_{i \in \mathbf{N}}^{\vee} \psi_{i}(x) \doteq \bigoplus_{i \in \mathbf{N}}^{\vee}\left[a_{i} \oplus \psi_{i}^{\prime}(x)\right],
$$

where the $\psi_{i}^{\prime}$ are max-plus linear. We will refer to this as a min-max basis expansion, or simply a min-max expansion, and we think of the set of such $\psi_{i}^{\prime}$ as a min-max basis for the space. The max-plus analog of this concept consists of maxplus vector spaces (more typically referred to as moduloids [3] or as idempotent semimodules [5], [10]) and max-plus basis expansions.

Now we indicate the complexity reduction problem of the previous section in a general form. Suppose we are given $f: \mathcal{X} \rightarrow \mathbb{R}$ with representation

$$
f(x)=\bigoplus_{m \in \mathcal{M}}^{\vee} t_{m}(x)=\min _{m \in \mathcal{M}} t_{m}(x)=\min _{m \in] 1, M[} t_{m}(x),
$$

where $\mathcal{X}$ will be a partially ordered vector space. Except where noted, we will take $\mathcal{X}=\mathbb{R}^{I}$ for clarity. We are looking for $\left\{a_{n}: \mathcal{X} \rightarrow \mathbb{R} \mid n \in\right] 1, N[\}$ with $N<M$, such that

$$
g(x) \doteq \bigoplus_{n \in \mathcal{N}}^{\vee} a_{n}(x)=\min _{n \in \mathcal{N}} a_{n}(x)=\min _{n \in] 1, N[} a_{n}(x)
$$

approximates $f(x)$ from above. Note that throughout the paper, we will let $\mathcal{M}=] 1, M[=\{1,2, \ldots M\}, \mathcal{N}=] 1, N[$ and $\mathcal{I}=] 1, I[$.

\section{A. Min-max spaces}

As indicated earlier, it is well-known that it is useful to apply max-plus basis expansions to solve certain HJB PDEs and their corresponding control problems. In particular, the solutions are represented as max-plus sums of affine or quadratic functions. In fact, the spaces of standard-sense convex and semiconvex functions have max-plus bases (more properly, max-plus spanning sets) consisting of linear and quadratic functions, respectively,

We will be applying the analogous concept, where the standard algebra will be replaced by the max-plus, and the max-plus will be replaced by the min-max. On $\mathbb{R}^{I}$, we will define the partial order $x \preceq y$ if $x_{i} \leq y_{i}$ for all $i \in \mathcal{I}$. Let $\mathcal{O}^{I}$ denote the closed first octant, i.e., $\mathcal{O}^{I}=[0, \infty)^{I} \doteq\{x \in$ $\left.\mathbb{R}^{I} \mid x \geq 0\right\}$. For $\delta \in \mathcal{O}^{I}$, let $\|\delta\|^{\oplus} \doteq \max _{i \in \mathcal{I}} \delta_{i}=\bigoplus_{i \in \mathcal{I}} \delta_{i}$. Let 1 denote a generic-length vector all of whose elements are 1's. Let

$$
\begin{gathered}
\mathcal{S}^{\mathbf{1}}\left(\mathbb{R}^{I}\right) \doteq\left\{f: \mathbb{R}^{I} \rightarrow \overline{\mathbb{R}} \mid \begin{array}{c}
0 \leq f(x+\delta)-f(x) \leq\|\delta\|^{\oplus}, \\
\left.\forall x \in \mathbb{R}^{I}, \delta \in \mathcal{O}^{I}\right\},
\end{array}\right.
\end{gathered}
$$

and

$$
\begin{gathered}
\mathcal{S}^{o, \mathbf{1}}\left(\mathbb{R}^{I}\right) \doteq\left\{f: \mathbb{R}^{I} \rightarrow \overline{\mathbb{R}} \mid \exists \varepsilon=\varepsilon(f)>0\right. \text { s.t. } \\
\left.0 \leq f(x+\delta)-f(x) \leq(1-\varepsilon)\|\delta\|^{\oplus}, \forall x \in \mathbb{R}^{I}, \delta \in \mathcal{O}^{I}\right\} .
\end{gathered}
$$

For $a \in \overline{\mathbb{R}}$ and $f, g \in \mathcal{S}^{\mathbf{1}}\left(\mathbb{R}^{I}\right)$, we define the inherited operations

$$
\begin{aligned}
& {\left[f \oplus^{\vee} g\right](x)=\min \{f(x), g(x)\} \quad \forall x \in \mathbb{R}^{I},} \\
& {\left[a \otimes^{\vee} f\right](x)=\max \{a, f(x)\} \quad \forall x \in \mathbb{R}^{I} .}
\end{aligned}
$$

Remark 3.1: In [25], $f: \mathbb{R}^{I} \rightarrow \overline{\mathbb{R}}$ is said to be subtopical or increasing plus-sub-homogeneous if $f$ is increasing according to partial order $\succeq$, and if

$$
f(x+\lambda \mathbf{1}) \leq f(x)+\lambda \quad \forall x \in \mathbb{R}^{I}, \forall \lambda>0 .
$$

Suppose $f \in \mathcal{S}^{1}\left(\mathbb{R}^{I}\right)$. Then, it is obvious that $f$ is subtopical. Suppose $f \notin \mathcal{S}^{1}\left(\mathbb{R}^{I}\right)$. Then, there exists $x \in \mathbb{R}^{I}$ and $\delta \succeq 0$ such that

$$
f(x+\delta)>f(x)+\|\delta\|^{\oplus} .
$$

Let $\bar{\delta} \doteq\|\delta\|^{\oplus}$. Then,

$$
f(x+\bar{\delta} \mathbf{1}) \geq f(x+\delta)>f(x)+\|\delta\|^{\oplus},
$$

which implies that $f$ is not sub-topical. Therefore, $\mathcal{S}^{\mathbf{1}}\left(\mathbb{R}^{I}\right)$ is exactly the space of sub-topical functions from $\mathbb{R}^{I}$ to $\overline{\mathbb{R}}$.

It is also useful to note the following easily obtained result.

Proposition 3.2: Suppose $f \in \mathcal{S}^{1}\left(\mathbb{R}^{I}\right)$. Suppose there exists $x \in \mathbb{R}^{I}$ such that $f(x)=+\infty$ [resp., $-\infty$ ]. Then, $f(y)=+\infty$ [resp., $-\infty$ ] for all $y \in \mathbb{R}^{I}$.

Consequently, excepting two special cases $f=+\infty$ and $f=-\infty$, we can assume any $f \in \mathcal{S}^{1}$ is everywhere finite. Except where there is some difficulty or interesting phenomenon, we will henceforth ignore these two special cases.

We will refer to a space as a min-max vector space if it satisfies the standard conditions (c.f. [11]). The definition of a max-plus vector space is analogous.

Theorem 3.3: $\mathcal{S}^{\mathbf{1}}$ and $\mathcal{S}^{o, 1}$ are min-max vector spaces.

Remark 3.4: It is not difficult to show that if $f \in \mathcal{S}^{\mathbf{1}}$ (and so, in $\left.\mathcal{S}^{o, \mathbf{1}}\right)$, and there exists $x \in \mathbb{R}^{I}$ such that $f(x)=+\infty$ then $f(y)=+\infty$ for all $y \in \mathbb{R}^{I}$. Similarly, if there exists $x \in \mathbb{R}^{I}$ such that $f(x)=-\infty$ then $f(y)=-\infty$ for all $y \in \mathbb{R}^{I}$.

There are obvious generalizations of these spaces. These may be useful for classes of control and game problems whose solutions do not naturally fall in the above two spaces. However, we leave that to later efforts.

One of the most useful aspects of looking at the spaces of convex and semiconvex spaces as max-plus vector spaces 
was that these spaces had countable max-plus bases. For example the space of convex functions has the set of (standardalgebra) linear functionals with rational coefficients as a countable max-plus basis. We are interested in analogous results here, that is, for any $f \in \mathcal{S}^{o, 1}\left(\mathbb{R}^{I}\right)$, we would like to represent it in terms of the coefficients in its min-max basis expansion. (Again, note that our use of the term "basis expansion" in this context is non-standard.) Specifically, we would like $\left\{\phi_{k}\right\}_{k \in \mathbf{N}} \subset \mathcal{S}^{\mathbf{1}}\left(\mathbb{R}^{I}\right)$ such that, given $f \in$ $\mathcal{S}^{\mathbf{1}}\left(\mathbb{R}^{I}\right)$, there exists $\left\{c_{k}\right\}_{k \in \mathbf{N}} \subset \overline{\mathbb{R}}$ such that

$$
f=\bigoplus_{k \in \mathbf{N}}^{\vee} c_{k} \otimes^{\vee} \phi_{k}
$$

i.e., for all $x \in \mathbb{R}^{I}$,

$$
\begin{aligned}
f(x) & =\left[\bigoplus_{k \in \mathbf{N}}^{\vee} c_{k} \otimes^{\vee} \phi_{k}\right](x) \\
& =\inf _{k \in \mathbf{N}} \max \left\{c_{k}, \phi_{k}(x)\right\} .
\end{aligned}
$$

We were only able to obtain this result for $f \in \mathcal{S}^{o, 1}\left(\mathbb{R}^{I}\right)$. We will also be interested in finding the best approximation given that we can use at most $N<\infty$ such $\phi_{k}$. A particular subclass of this second problem will be our main focus in the paper.

Returning to the first issue, we take $\psi(x, z): \mathbb{R}^{I} \times \mathbb{R}^{I} \rightarrow$ $I R$ to be

$$
\psi(x, z) \doteq z \odot x \doteq \bigoplus_{i \in \mathcal{I}} z_{i} \otimes x_{i}=\max _{i \in \mathcal{I}}\left\{z_{i}+x_{i}\right\} .
$$

We may think of $\psi(\cdot, z)$ as a max-plus linear function with "slope", $z$. We will be taking $\phi_{k}(x)=\psi\left(x, z^{k}\right)$ where the $z^{k}$ will form a countable dense subset of $\mathbb{R}^{I}$. The result will follow if we have

$f(x)=\int_{\mathbb{R}^{I}}^{\oplus^{\vee}} c(z) \otimes^{\vee} \psi(x, z) d z \inf _{z \in \mathbb{R}^{I}}\{\max [c(z), \psi(x, z)]\}$,

where $c$ has sufficient continuity properties. Note that this would imply that $f$ was the lower envelope of a set of functions. Further, note that

$$
c(z) \otimes^{\vee} \psi(x, z)=c(z) \oplus \psi(x, z)
$$

where the $\psi(\cdot, z)$ are max-plus linear. In other words, $f$ would be an infimum of max-plus affine functions.

Remark 3.5: Alternatively, we would say that, with $\tilde{f}(x) \doteq-f(x)$ for all $x, \tilde{f}$ would be the upper envelope of a set of max-plus affine functions. This would correspond to the notion of "abstract convexity" [24], [25], where the standard affine functions are replaced by max-plus affine functions. See also [6] in this regard.

We now fill in the details of this plan for min-max basis representations. First we obtain what may be part of a duality result.

Theorem 3.6: Let $f \in \mathcal{S}^{o, 1}\left(\mathbb{R}^{I}\right)$. Then,

$$
f(x)=\int_{\mathbb{R}^{I}}^{\oplus^{\vee}} c(z) \otimes^{\vee} \psi(x, z) d z \quad \forall x \in \mathbb{R}^{I}
$$

where

$$
\begin{aligned}
c(z) & =\sup \left\{f(x) \mid \psi(x, z)-f(x) \leq 0 \forall x \in \mathbb{R}^{I}\right\} \\
& =-\int_{\psi(x, z) \otimes^{\vee} f(x) \leq f(x)}^{\oplus^{\vee}}-f(x) d x \quad \forall z \in \mathbb{R}^{I} .
\end{aligned}
$$

Proof: Suppose $f \neq \pm \infty$; otherwise the proof is trivial. Suppose $z$ is such that $\psi(x, z)-f(x)<0$. Then, by definition, $c(z) \geq f(x)$. Consequently, $c(z) \otimes^{\vee} \psi(x, z) \geq$ $f(x)$ for any $z \in \mathbb{R}^{I}$, and so

$$
\int_{\mathbb{R}^{I}}^{\oplus^{\vee}} c(z) \otimes^{\vee} \psi(x, z) d z \geq f(x) .
$$

Now, we obtain the reverse inequality. Let $\tilde{z}=\tilde{z}(x)$ be given by

$$
[\tilde{z}]_{i}=f(x)-x_{i} \quad \forall i \in \mathcal{I}
$$

Then,

$$
\psi(x, \tilde{z})=\tilde{z} \odot x=\max _{i \in \mathcal{I}}\left[f(x)-x_{i}+x_{i}\right]=f(x) .
$$

Suppose $c(\tilde{z})>f(x)$. This implies there exists $y \in \mathbb{R}^{I}$ such that

$$
\begin{aligned}
& f(y)>f(x), \\
& \psi(y, \tilde{z}) \leq f(y) .
\end{aligned}
$$

Let $\mathcal{I}^{\prime} \doteq\left\{i \in \mathcal{I} \mid y_{i}>x_{i}\right\}$. By (21) and the fact that $f \in \mathcal{S}^{\mathbf{1}}$, one finds that $\mathcal{I}^{\prime} \neq \emptyset$. Also,

$$
\begin{aligned}
\psi(x, \tilde{z}) & =\max _{i \in \mathcal{I}}\left[f(x)+\left(y_{i}-x_{i}\right)\right] \\
& =f(x)+\max _{i \in \mathcal{I}^{\prime}}\left[y_{i}-x_{i}\right]
\end{aligned}
$$

Now, $f \in \mathcal{S}^{o, 1}$ implies that there exists $\varepsilon>0$ such that

$$
f(y)-f(x) \leq(1-\varepsilon) \max _{i \in \mathcal{I}^{\prime}}\left[y_{i}-x_{i}\right]
$$

Combining (23) and (24), one has

$$
\psi(y, \tilde{z}) \geq f(y)+\varepsilon \max _{i \in \mathcal{I}^{\prime}}\left[y_{i}-x_{i}\right]
$$

which, since $\mathcal{I}^{\prime} \neq \emptyset$,

$$
>f(y),
$$

which contradicts (22). Therefore,

$$
c(\tilde{z}) \leq f(x) .
$$

Then, by (20) and (25),

$$
c(\tilde{z}) \otimes^{\vee} \psi(x, \tilde{z})=f(x) .
$$

Combining (19) and (26) yields the result.

Now define, for any $z \in \mathbb{R}^{I}$,

$$
A_{z} \doteq\left\{x \in \mathbb{R}^{I} \mid \psi(x, z)-f(x) \leq 0\right\},
$$

and note

$$
c(z)=\sup \left\{f(x) \mid x \in A_{z}\right\} .
$$

In the next results, we will be obtaining a continuity property on $c(\cdot)$. This will allow us to obtain our countable min-max basis for $\mathcal{S}^{o, 1}$. 
Lemma 3.7: Let $f \in \mathcal{S}^{o, 1}\left(\mathbb{R}^{I}\right), f \neq \pm \infty$. Let $z \in \mathbb{R}^{I}$, and $\bar{a} \in \mathbb{R}$. There exists $M=M(z, \bar{a}, f)$ such that $x_{i} \leq M$ for all $i \in \mathcal{I}$ and all $x \in \mathbb{R}^{I}$ such that $\psi(x, z)-f(x) \leq \bar{a}$.

The next lemma follows by taking $\bar{a}=0$ in Lemma 3.7.

Lemma 3.8: Let $f \in \mathcal{S}^{o, 1}\left(\mathbb{R}^{I}\right), f \neq \pm \infty$. Let $z \in \mathbb{R}^{I}$. There exists $M=M(z, f)$ such that $x_{i} \leq M$ for all $i \in \mathcal{I}$ and all $x \in A_{z}$.

Lemma 3.9: Let $f \in \mathcal{S}^{o, 1}\left(\mathbb{R}^{I}\right), f \neq \pm \infty$. Let $z \in \mathbb{R}^{I}$. Then, there exists $\hat{x} \in A_{z}$ such that $\hat{x} \succeq x$ for all $x \in A_{z}$ and $\psi(\hat{x}, z)-f(\hat{x})=0$.

Theorem 3.10: Let $f \in \mathcal{S}^{o, 1}, f \neq \pm \infty$. Let $z \in \mathbb{R}^{I}$. Let $z \in \mathbb{R}^{I}$. Then, there exists $\hat{x} \in A_{z}$ such that

$$
\hat{x} \succeq x \quad \forall x \in A_{z},
$$

$\psi(\hat{x}, z)-f(\hat{x})=0$ and $f(\hat{x})=c(z)$. Further, $\hat{x}$ is the unique element of $A_{z}$ satisfying (28).

Henceforth, we denote this $\hat{x}$ as $\hat{x}^{z}=\hat{x}^{z}\left(A_{z}\right)$.

Lemma 3.11: Let $f \in \mathcal{S}^{o, 1}, f \neq \pm \infty$. Let $z \in \mathbb{R}^{I}$. Then, $\hat{x}_{i}^{z}+z_{i}=f\left(\hat{x}^{z}\right)$ for all $i \in \mathcal{I}$.

Lemma 3.12: Let $f \in \mathcal{S}^{o, 1}$. Let $z \in \mathbb{R}^{I}$, and $\alpha \in \mathbb{R}^{I}$ with $\alpha \succeq 0$. Then, $c(z) \leq c(z-\alpha)$.

Theorem 3.13: Let $f \in \mathcal{S}^{o, 1}$. For any $z \in \mathbb{R}^{I}, c(z)=$ $\lim _{\alpha \downarrow 0} c(z-\alpha)$.

Proof: From Lemma 3.12, for $\alpha \succeq 0, c(z-\alpha) \geq c(z)$. We will show that it cannot be too much larger. We skip the trivial cases $f= \pm \infty$.

Let $\alpha \succeq 0$. Since $f \in \mathcal{S}^{o, 1}$ and $A_{z} \subseteq A_{z-\alpha}$, this implies

$$
f\left(\hat{x}^{z-\alpha}\right) \geq f\left(\hat{x}^{z}\right) .
$$

Further, by Theorem 3.10,

$\hat{x}_{i}^{z}+z_{i}-f\left(\hat{x}^{z}\right)=0 \quad$ and $\quad \hat{x}_{i}^{z-\alpha}+z_{i}-\alpha_{i}-f\left(\hat{x}^{z-\alpha}\right)=0$

for all $i \in \mathcal{I}$, and so

$$
\hat{x}_{i}^{z-\alpha}=\hat{x}_{i}^{z}+\alpha_{i}+f\left(\hat{x}^{z-\alpha}\right)-f\left(\hat{x}^{z}\right),
$$

which by (29) and the choice of $\alpha$,

$$
\geq \hat{x}_{i}^{z} \quad \forall i \in \mathcal{I} \text {. }
$$

Now, by (31) and the fact that $f \in \mathcal{S}^{o, 1}$,

$$
f\left(\hat{x}^{z-\alpha}\right)-f\left(\hat{x}^{z}\right) \leq(1-\varepsilon) \max _{i \in \mathcal{I}}\left[\hat{x}_{i}^{z-\alpha}-\hat{x}_{i}^{z}\right],
$$

which by (30),

$$
=(1-\varepsilon)\left\{f\left(\hat{x}^{z-\alpha}\right)-f\left(\hat{x}^{z}\right)+\max _{i \in \mathcal{I}}\left[\alpha_{i}\right]\right\} .
$$

This implies

$$
f\left(\hat{x}^{z-\alpha}\right) \leq f\left(\hat{x}^{z}\right)+\frac{1-\varepsilon}{\varepsilon} \max _{i \in \mathcal{I}}\left[\alpha_{i}\right],
$$

which, by Theorem 3.10, implies

$$
c(z-\alpha) \leq c(z)+\frac{1-\varepsilon}{\varepsilon} \max _{i \in \mathcal{I}}\left[\alpha_{i}\right]
$$

We are now ready to prove our countable basis result.
Theorem 3.14: Let $\left\{z_{k}\right\}_{k \in \mathbf{N}}$ be a countable dense subset of $\mathbb{R}^{I}$. Let $\phi_{k}(x) \doteq \psi\left(x, z_{k}\right)$ for all $x \in \mathbb{R}^{I}$ and all $k \in \mathbf{N}$. For any $f \in \mathcal{S}^{o, 1}$,

$$
f(x)=\bigoplus_{k \in \mathbf{N}}^{\vee} c_{k} \otimes^{\vee} \phi_{k}(x) \quad \forall x \in \mathbb{R}^{I},
$$

where

$$
c_{k} \doteq \sup \left\{f(x) \mid \psi\left(x, z_{k}\right)-f(x) \leq 0 \forall x \in \mathbb{R}^{I}\right\}
$$

for all $k \in \mathbf{N}$.

Proof: Let $x \in \mathbb{R}^{I}$ and $\varepsilon>0$. By Theorem 3.6, there exists $\bar{z} \in \mathbb{R}^{I}$ such that

$f(x) \leq c(\bar{z}) \otimes^{\vee} \psi(x, \bar{z})=\max \{c(\bar{z}), \psi(x, \bar{z})\} \leq f(x)+\varepsilon / 2$.

By the density of $\left\{z_{k}\right\}_{k \in \mathbf{N}}$, Theorem 3.13 and the continuity of $\psi$, there exists $\bar{k} \in \mathbf{N}$ such that

$$
c(\bar{z}) \leq c\left(z_{\bar{k}}\right) \leq c(\bar{z})+\varepsilon / 2
$$

and

$$
\left|\psi(x, \bar{z})-\psi\left(x, z_{\bar{k}}\right)\right| \leq \varepsilon / 2 .
$$

Combining (34)-(36), we have

$$
f(x) \leq c_{\bar{k}} \otimes^{\vee} \phi_{\bar{k}}(x) \leq f(x)+\varepsilon .
$$

Since this is true for all $\varepsilon>0$, we are done.

\section{B. $\mathcal{S}^{o, 1}$ and max-plus convexity}

In [13], a problem similar to that described above was formulated, but in that case the max-plus algebra was replaced by the standard field, and the min-max algebra was replaced by the max-plus algebra. In solving that problem, we used a certain optimization criterion which was convex and increasing. Below, we will use a similar technique. Consequently, we will be dealing here with max-plus convex functions; the optimization criterion will be max-plus convex. As the min-max algebra suggests a natural order on the range space, $\bar{R}$, which is the opposite of the standard order, this will lead us to a different definition of max-plus convex functions from that used elsewhere (c.f. [6], [25]).

We begin with the definition of max-plus convex sets. A set, $\mathcal{C} \subseteq \mathbb{R}^{I}$ is max-plus convex if

$$
\lambda_{1} \otimes x^{1} \oplus \lambda_{2} \otimes x^{2} \in \mathcal{C}
$$

for all $x^{1}, x^{2} \in \mathcal{C}$ and all $\lambda_{1}, \lambda_{2} \in[-\infty, 0]$ such that $\lambda_{1} \oplus$ $\lambda_{2}=0$. See [6], [25]. We now turn to max-plus convex functions. We would like the set of such functions to form a min-max vector space. Consequently, we define the ordering on the range space, $\overline{\mathbb{R}}$, by $y_{1} \preceq^{R} y_{2}$ if $y_{1} \geq y_{2}$, and $y_{1} \prec^{R} y_{2}$ if $y_{1}>y_{2}$; relations $\succeq^{R}$ and $\succ^{R}$ are defined analogously. We henceforth refer to this as the range order. Suppose $f$ : $\mathbb{R}^{I} \rightarrow \overline{\mathbb{R}}$, and define the max-plus epigraph as

$$
\mathrm{epi}^{\oplus} f \doteq\left\{(x, y) \in \mathbb{R}^{I} \times \overline{\mathbb{R}} \mid y \succeq^{R} f(x)\right\} .
$$

Note that epi ${ }^{\oplus} f$ lies below the graph of $f$ in the standard sense. Alternatively, $f$ may be referred to as the hypograph [25], but due to the natural reversal of order in the range 
space here, the term max-plus epigraph is more appropriate in this context. Lastly, we say $f$ is max-plus convex if epi $^{\oplus} f$ is max-plus convex. We now show that functions formed by min-max integrals (infima) of max-plus affine functions are max-plus convex.

Theorem 3.15: Let $Z \subseteq \mathbb{R}^{I}, c: Z \rightarrow \overline{\mathbb{R}}$, and

$$
f(x) \doteq \int_{Z}^{\oplus^{\vee}} c(z) \oplus \psi(x, z) d z=\inf _{z \in Z}\{c(z) \oplus x \odot z\}
$$

for all $x \in \mathbb{R}^{I}$. Then $f$ is max-plus convex.

Corollary 3.16: Suppose $f(x)=\bigoplus_{k \in \mathcal{K}}^{\vee} c_{k} \oplus z^{k} \odot x$ for all $x$, where $\mathcal{K} \subseteq \mathbf{N}$, and $c_{k} \in \overline{\mathbb{R}}$ and $z^{k} \in \mathbb{R}^{I}$ for all $k \in \mathcal{K}$. Then, $f$ is max-plus convex.

Corollary 3.17: If $f \in \mathcal{S}^{o, 1}$, then $f$ is max-plus convex.

Theorem 3.18: If $f$ is max-plus convex, then $f \in \mathcal{S}^{1}$.

\section{Complexity Reduction And Abstract FORMULATION}

Recall that our originating problem was complexity reduction in a min-max expansion. That is, given $f: G \subseteq \mathcal{X} \rightarrow \mathbb{R}$ with representation

$$
f(x)=\bigoplus_{m \in \mathcal{M}}^{\vee} t_{m}(x)=\min _{m \in \mathcal{M}} t_{m}(x),
$$

we are looking for a reduced complexity expansion

$$
g(x)=\bigoplus_{n \in \mathcal{N}}^{\vee} a_{n}(x)=\min _{n \in \mathcal{N}} a_{n}(x)
$$

approximating $f$ as "well" as possible from above. The $a_{n}$ and $t_{m}$ will be selected from a specified class of function, such as affine functions. We take $\mathcal{X} \doteq \mathbb{R}^{I}$ throughout. We will use a measure of approximation quality which is monotonic (in a sense to be specified) and max-plus convex. Specifically, we wish to minimize

$$
\begin{aligned}
J\left(\left\{a_{n}\right\}_{n \in \mathcal{N}}\right) & =\int_{G}^{\oplus}\left\{\left[\bigoplus_{n \in \mathcal{N}}^{\vee} a_{n}(x)\right]-\left[\bigoplus_{m \in \mathcal{M}}^{\vee} t_{m}(x)\right]\right\} d x \\
& =\sup _{x \in G}\left\{\min _{n \in \mathcal{N}} a_{n}(x)-\min _{m \in \mathcal{M}} t_{m}(x)\right\}
\end{aligned}
$$

conditioned on

$$
\bigoplus_{n \in \mathcal{N}}^{\vee} a_{n}(x) \geq \bigoplus_{m \in \mathcal{M}}^{\vee} t_{m}(x) \quad \forall x \in G,
$$

or equivalently, conditioned on

$$
a_{n}(x) \geq \bigoplus_{m \in \mathcal{M}}^{\vee} t_{m}(x) \quad \forall x \in G, \forall n \in \mathcal{N} .
$$

\section{A. Abstract formulation}

We consider an abstract problem which will subsume (39),(40), and prove the main result for this abstract problem here. Further below, we will demonstrate that (39),(40) is indeed subsumed by the following problem.

Let $\mathcal{Y}$ be a max-plus vector space with partial order $\preceq^{\oplus}$. Given $p \in \mathcal{Y}$, define the downward cone of $p$ to be

$$
\mathcal{D}(p)=\left\{q \in \mathcal{Y} \mid q \preceq^{\oplus} p\right\} .
$$

For any $K \in \mathbf{N}$, let

$$
S^{\oplus K} \doteq\left\{\lambda=\left\{\lambda_{k}\right\}_{k \in] 1, K[} \in[-\infty, 0]^{K} \mid \bigoplus_{k \in] 1, K[} \lambda_{k}=0\right\} .
$$

Given $P \subseteq \mathcal{Y}$, let $\langle P\rangle^{\oplus}$ denote the max-plus convex hull of $P$. That is,

$$
\begin{aligned}
\langle P\rangle^{\oplus} \doteq\{q \in \mathcal{Y} \mid & \exists \widehat{N} \in \mathbf{N},\left\{\lambda_{k}\right\}_{k=1}^{\widehat{N}} \subset S^{\oplus \widehat{N}} \text { and } \\
& \left.\left\{p^{k}\right\}_{k=1}^{\widehat{N}} \subseteq P \text {, s.t. } q=\bigoplus_{k=1}^{\widehat{N}} \lambda_{k} \otimes p^{k}\right\} .
\end{aligned}
$$

Finally, given $P \subseteq \mathcal{Y}$, define the max-plus cornice of $P$ to be

$$
\mathcal{C}^{\oplus}(P)=\bigcup_{q \in\langle P\rangle \oplus} \mathcal{D}(q) .
$$

We extend these definitions to product spaces. We define the inherited (component-wise) partial order on $\mathcal{Y}^{N}$ as follows. Let $P, Q \in \mathcal{Y}^{N}$, where $P=\left\{p^{i}\right\}_{i \in \mathcal{N}}$ and $Q=$ $\left\{q^{i}\right\}_{i \in \mathcal{N}}$. Then $Q \preceq^{\oplus} P$ if $q^{i} \preceq^{\oplus} p^{i}$ for all $\left.i \in \mathcal{N}=\right] 1, N[$. We will be abusing notation slightly by letting $P \in \mathcal{Y}^{N}$ also denote a set of $N$ elements of $\mathcal{Y}$ (Otherwise, one may define a mapping from elements of $\mathcal{Y}^{N}$ to subsets of $\mathcal{Y}$ of size $N$.) Prior to obtaining the abstract-formulation result, we obtain a simple equivalence.

Lemma 4.1: Let $\mathcal{P} \subseteq \mathcal{Y}$. Then

$$
\left[\langle\mathcal{P}\rangle^{\oplus}\right]^{N}=\left\langle\mathcal{P}^{N}\right\rangle^{\oplus}
$$

where the $N$ superscripts indicate outer product $N$-times, i.e., $P \in \mathcal{P}^{N}$ if $P=\left\{p^{i}\right\}_{i \in \mathcal{N}}$ where $p^{i} \in \mathcal{P}$ for all $i$.

Now, given $\mathcal{P} \subseteq \mathcal{Y}$, define the $N$-dimensional outerproduct max-plus cornice of $P$ to be

$$
\begin{aligned}
\mathcal{C}^{\oplus N}(\mathcal{P}) & \doteq\left[\mathcal{C}^{\oplus}(\mathcal{P})\right]^{N} \\
& =\left\{Q=\left\{q^{i}\right\}_{i \in \mathcal{N}} \in \mathcal{Y}^{N} \mid q^{i} \in \mathcal{C}^{\oplus}(\mathcal{P}) \forall i \in \mathcal{N}\right\} .
\end{aligned}
$$

We will say that $J: \mathcal{Y}^{N} \rightarrow \overline{\mathbb{R}}$ is monotonically increasing, relative to the inherited partial order and range order, $\preceq^{R}$, if $Q \preceq^{\oplus} P$ implies $J(Q) \preceq^{R} J(P)$. We can now state and prove our abstract result.

Theorem 4.2: Let $\mathcal{Y}$ be a max-plus vector space with partial order, $\preceq^{\oplus}$. Let $\mathcal{P}=\left\{p^{m}\right\}_{m \in \mathcal{M}} \subset \mathcal{Y}$. Suppose $N<M$. Suppose $J: \mathcal{Y}^{N} \rightarrow \mathbb{R}$ is monotonically increasing (relative to the inherited partial order and range order) and max-plus convex on $\mathcal{C}^{\oplus}(\mathcal{P})$. Then, there exists $\bar{Q}=\left\{\bar{q}^{i}\right\}_{i \in \mathcal{N}}$ with $\bar{q}^{i} \in \mathcal{P}$ for all $i \in \mathcal{N}$, such that

$$
\begin{aligned}
J(\bar{Q})=J^{*} & \doteq \max ^{R}\left\{J(Q) \mid Q \in \mathcal{C}^{\oplus^{N}}(\mathcal{P})\right\} \\
& =\min \left\{J(Q) \mid Q \in \mathcal{C}^{\oplus^{N}}(\mathcal{P})\right\} .
\end{aligned}
$$

where for $Z \subset \bar{R}, \max ^{R} Z \doteq \min Z$.

Proof: Let $\varepsilon>0$. Let $Q=\left\{q_{i}\right\}_{i \in \mathcal{N}} \in \mathcal{C}^{\oplus^{N}}(\mathcal{P})$ be such that $J(Q) \succ^{R} J^{*}-\varepsilon$. Since $Q \in \mathcal{C}^{\oplus}(\mathcal{P})$, there exists $Q^{u}=\left\{q_{i}^{u}\right\}_{i \in \mathcal{N}}$ such that

$$
Q^{u} \in\left[\langle\mathcal{P}\rangle^{\oplus}\right]^{N}
$$


and $Q \preceq{ }^{\oplus} Q^{u}$. Then, by the monotonicity of $J$,

$$
J\left(Q^{u}\right) \succeq^{R} J(Q) \succeq^{R} J^{*}-\varepsilon .
$$

By (41) and Lemma 4.1, $Q^{u} \in\left\langle\mathcal{P}^{N}\right\rangle^{\oplus}$. Consequently, there exists $K<\infty,\left\{\lambda_{k} \mid k \in\right] 1, K[\} \in S^{\oplus K}$ and $\widehat{P}^{k} \in \mathcal{P}^{N}$ for $k \in] 1, K[$ such that

$$
Q^{u}=\bigoplus_{k \in] 1, K[} \lambda_{k} \otimes \widehat{P}^{k}
$$

By (43), the max-plus convexity of $J$, and the fact that $\left\{\lambda_{k} \mid k \in\right] 1, K[\} \in S^{\oplus K}$,

$$
J\left(Q^{u}\right) \preceq^{R} \bigoplus_{k \in] 1, K[} \lambda_{k} \otimes J\left(\widehat{P}^{k}\right) \preceq{ }^{R} \sup ^{R}{ }_{\widehat{P} \in \mathcal{P}^{N}} J(\widehat{P}),
$$

where for $Z \subseteq \bar{R}, \sup ^{R} Z=\inf Z$. Now, by (42) and (44),

$$
\sup ^{R}{\widehat{P} \in \mathcal{P}^{N}} J(\widehat{P}) \succeq^{R} J^{*}-\varepsilon .
$$

Since this is true for all $\varepsilon>0$,

$$
\sup _{\widehat{P} \in \mathcal{P}^{N}}^{R} J(\widehat{P}) \succeq^{R} J^{*} .
$$

However, $\mathcal{P}^{N}$ is a finite set. Therefore, the range-order supremum is attained at some $\bar{Q} \in \mathcal{P}^{N}$, and so $J(\bar{Q}) \succeq^{R} J^{*}$.

\section{MaX-plus AfFine Functionals}

Recall that we are interested in min-max expansions of max-plus convex functionals. More specifically, we are interested in the special case of reduced-complexity expansions of finite min-max sums of max-plus affine functionals. We will reduce this problem to the abstract formulation of the previous section, and apply Theorem 4.2 to show that pruning is optimal.

Our problem is to minimize, i.e., range-order maximize, criterion $J$ of (39) subject to constraints (40), where now the $t_{m}$ and $a_{n}$ functionals will be max-plus affine functions. More specifically, we range-order maximize (minimize)

$$
J(A) \doteq \int_{G}^{\oplus}\left\{\left[\bigoplus_{n \in \mathcal{N}}^{\vee} \alpha^{n} \odot x\right]-\left[\bigoplus_{m \in \mathcal{M}}^{\vee} \tau^{m} \odot x\right]\right\} d x
$$

where $A \doteq\left\{\alpha^{n}\right\}_{n \in \mathcal{N}}$, subject to the constraints

$$
\alpha^{n} \cdot x \geq \bigoplus_{m \in \mathcal{M}}^{\vee} \tau^{m} \odot x \quad \forall x \in \mathbb{R}^{I}, \forall n \in \mathcal{N} .
$$

Let $T \doteq\left\{\tau^{m}\right\}_{m \in \mathcal{M}}$, and let

$$
\begin{aligned}
\widehat{\mathcal{C}}(T) & \doteq\left\{\alpha \in \mathbb{R}^{I} \mid \alpha \odot x \preceq^{R} \bigoplus_{m \in \mathcal{M}}^{\vee} \tau^{m} \odot x \forall x \in \overline{\mathbb{R}}^{I}\right\} \\
& =\left\{\alpha \in \mathbb{R}^{I} \mid \alpha \odot x \geq \bigoplus_{m \in \mathcal{M}}^{\vee} \tau^{m} \odot x \forall x \in \overline{\mathbb{R}}^{I}\right\} .
\end{aligned}
$$

We want to show that $\widehat{\mathcal{C}}(T)$ is the max-plus cornice of $T$. That is, we want to show $\widehat{\mathcal{C}}(T)=\mathcal{C}(T)$.

We say that a set, $\mathcal{P} \subseteq \mathbb{R}^{I}$ is (range-order) downward if $p \in \mathcal{P}, q \preceq^{R} p$ implies $q \in \mathcal{P}$, where $q \preceq^{R} p$ if $q_{i} \preceq^{R} p_{i}$ for all $i \in \mathcal{I}$. (Of course, this is upward in the normal sense.)
Note that the range-order of the previous section will now be carrying over to the coefficients in the linear functionals here.

The following series of lemmas is easily obtained.

Lemma 5.1: (ordering product-invariance) Let $K \in \mathbf{N}$ and $a, b, c \in \overline{\mathbb{R}}^{K}$, with $a{ }^{R} b$. Then, $c \odot a{ }^{R} c \odot b$.

Lemma 5.2: $\widehat{\mathcal{C}}(T)$ is range-order downward.

Lemma 5.3: $\widehat{\mathcal{C}}(T)$ is max-plus convex.

Lemma 5.4: For any $\mathcal{P} \subseteq \overline{\mathbb{R}}^{I}, \mathcal{C}^{\oplus}(\mathcal{P})$ is range-order downward.

We do not include a proof of the following.

Lemma 5.5: For any $\mathcal{P} \subseteq \mathbb{R}^{I},\langle\mathcal{P}\rangle^{\oplus}$ is max-plus convex.

Lemma 5.6: For any $\mathcal{P} \subseteq \bar{R}^{I}, \mathcal{C}^{\oplus}(\mathcal{P})$ is max-plus convex.

The next lemma is obvious.

Lemma 5.7: For any $\mathcal{P} \subseteq \overline{\mathbb{R}}^{I}, \widehat{\mathcal{C}}(\mathcal{P}) \supseteq \mathcal{P}$ and $\mathcal{C}^{\oplus}(\mathcal{P}) \supseteq$ $\mathcal{P}$.

Note that we have now shown that for any $\mathcal{P} \subseteq \overline{\mathbb{R}}^{I}$, both $\widehat{\mathcal{C}}\left(\mathcal{P}\right.$ and $\mathcal{C}^{\oplus}(\mathcal{P})$ are range-order downward, max-plus convex and contain $\mathcal{P}$ We will show that they are identical.

Lemma 5.8: Let $\mathcal{P} \subseteq \overline{\mathbb{R}}^{I}$. Suppose $\mathcal{A} \subseteq \overline{\mathbb{R}}^{I}$ is rangeorder downward, max-plus convex and such that $\mathcal{P} \subseteq \mathcal{A}$. Then, $\mathcal{C}^{\oplus}(\mathcal{P}) \subseteq \mathcal{A}$.

Lemma 5.9: $\mathcal{C}^{\oplus}(T) \subseteq \widehat{\mathcal{C}}(T)$.

Lemma 5.10: There exists $m \in \mathcal{M}$ such that $\alpha \preceq^{R} \tau^{m}$ if and only if $\alpha \in \mathcal{C}^{\oplus}(T)$.

Lemma 5.11: Suppose $\alpha \odot x \geq \min _{m \in \mathcal{M}} \tau^{m} \odot x$ for all $x \in \overline{\mathbb{R}}^{I}$. Then, there exists $m \in \mathcal{M}$ such that $\alpha \preceq^{R} \tau^{m}$ (i.e., $\alpha_{i} \geq \tau_{i}^{m}$ for all $\left.i \in \mathcal{I}\right)$.

Lemma 5.12: If $\alpha \in \widehat{\mathcal{C}}(T)$, then there exists $m \in \mathcal{M}$ such that $\alpha \preceq^{R} \tau^{m}$.

Lemma 5.13: $\widehat{\mathcal{C}}(T) \subseteq \mathcal{C}^{\oplus}(T)$.

We now have

Theorem 5.14: $\widehat{\mathcal{C}}(T)=\mathcal{C}^{\oplus}(T)$.

Recall that we want to minimize (range-order maximize) $J(A)$ given by (45) subject to $A=\left\{\alpha^{n}\right\}_{n \in \mathcal{N}}$ with $\alpha^{n} \in$ $\widehat{\mathcal{C}}(T)$ for all $n \in \mathcal{N}$. By Theorem 5.14, the constraint is equivalent to $\alpha^{n} \in \mathcal{C}^{\oplus}(T)$ for all $n \in \mathcal{N}$, or equivalently,

$$
A \in \mathcal{C}^{\oplus N}(T) .
$$

In order to apply Theorem 4.2 to problem (45)/(47), we need to show that $J$ has the required properties. We now proceed to do this.

Theorem 5.15: $J$ given by (45) is monotonically increasing (relative to the inherited partial order and the range-order) and max-plus convex on $\left[\bar{R}^{I}\right]^{N}$.

We are now in a position to state and prove our main result of the section.

Theorem 5.16: There exists $A^{*}$ minimizing (i.e., rangeorder maximizing) $J$ subject to constraints (46). Further, there exist $\left\{m_{n}\right\}_{n \in \mathcal{N}} \subset \mathcal{M}$ such that $A^{*}=\left\{\tau^{m_{n}}\right\}_{n \in \mathcal{N}}$.

Remark 5.17: Note that the above result covers only the max-plus linear case. We may extend this to the affine case on $G^{\prime} \subseteq \overline{\mathbb{R}}^{I^{\prime}}$ with $I^{\prime} \doteq I-1$ by letting $G=G^{\prime} \times\{0\}$. 
Then with $\alpha^{n}=\left(\left[\alpha^{\prime}\right]^{n}, \beta\right) \in \overline{\mathbb{R}}^{I}$, for any $x^{\prime} \in G^{\prime}$ there exists unique $x \in G$ given by $x=\left(x^{\prime}, 0\right)$ such that

$$
\left[\alpha^{\prime}\right]^{n} \odot x^{\prime} \oplus \beta=\alpha^{n} \odot\left(x^{\prime}, 0\right)=\alpha^{n} \odot x .
$$

With this equivalence, one extends our result to affine functionals.

The point of Theorem 5.16 is that the search for an optimal reduced-complexity approximation can be reduced to a pruning problem. That is, suppose we have a min-max approximate expansion of some max-plus convex function, $f \in \mathcal{S}^{o, \mathbf{1}}\left(\mathbb{R}^{I}\right)$, given by

$$
f\left(x^{\prime}\right) \simeq \bigoplus_{m \in \mathcal{M}}^{\vee} c^{m} \otimes^{\vee} \max _{i \in \mathcal{I}^{\prime}}\left\{z_{i}^{n}+x_{i}^{\prime}\right\}
$$

where $\left.\mathcal{I}^{\prime} \doteq\right] 1, I^{\prime}[$

$$
\begin{aligned}
& =\bigoplus_{m \in \mathcal{M}}^{\vee} \max \left\{c^{m}, m \max _{i \in \mathcal{I}^{\prime}}\left\{z_{i}^{n}+x_{i}^{\prime}\right\}\right\} \\
& \doteq \bigoplus_{m \in \mathcal{M}} \tau^{m} \odot\left(x^{\prime}, 0\right)=\bigoplus_{m \in \mathcal{M}} \vee \tau^{m} \odot x
\end{aligned}
$$

for $x \in G^{\prime} \times\{0\}=G$ with

$$
\tau_{i}^{m}= \begin{cases}z_{i}^{m} & i \leq I-1 \\ c^{m} & i=I .\end{cases}
$$

Furthermore, suppose we would like a lower-complexity approximation from above. (Note that if one goes "below" $f$ with an approximation, this can never be corrected by the addition of more terms to the approximation.) If we see $J$ as a measure of the quality of the approximation, then Theorem 5.16 tells us that we may find the best approximation merely through pruning of the set of $\tau^{m}$.

\section{Closing Remarks}

There are three main purposes to this paper. The first is to give a brief introduction to an idempotent curseof-dimensionality-free algorithm for discrete-time dynamic games. It is easily seen that the feasibility of that approach will rely on a means for complexity reduction of the solution representation at each step. That motivates the second theme of the paper, where this second theme is captured in the main result that the optimal complexity reduction is achieved by a pruning of the given set of max-plus affine functions. Lastly, this naturally suggests a discussion of the min-max spaces $\mathcal{S}^{\mathbf{1}}$ and $\mathcal{S}^{o, 1}$, and that is included. From a practical standpoint, the main point is the introduction of a new approach to the solution of dynamic games, and the related machinery.

\section{REFERENCES}

[1] M. Akian, S. Gaubert and A. Lakhoua, The max-plus finite element method for solving deterministic optimal control problems: basic properties and convergence analysis, SIAM J. Control and Optim., 47 (2008), 817-848.

[2] M. Akian, S. Gaubert and A. Lakhoua, A max-plus finite element method for solving finite horizon deterministic optimal control problems, Proc. $16^{t h}$ International Symposium on Mathematical Theory of Networks and Systems (2004).

[3] F.L. Baccelli, G. Cohen, G.J. Olsder and J.-P. Quadrat, Synchronization and Linearity, John Wiley, New York, 1992.

[4] R.A. Cuninghame-Green, Minimax Algebra, Lecture Notes in Economics and Mathematical Systems 166, Springer, New York, 1979.
[5] G. Cohen, S. Gaubert and J.-P. Quadrat, Duality and Separation Theorems in Idempotent Semimodules, Linear Algebra and Applications, 379 (2004), 395-422.

[6] G. Cohen, S. Gaubert, J.-P. Quadrat and I. Singer, Max-Plus Convex Sets and Functions, preprint.

[7] W.H. Fleming, Max-plus stochastic processes, Applied Math. and Optim., 49 (2004), 159-181.

[8] W.H. Fleming and W.M. McEneaney, A max-plus based algorithm for an HJB equation of nonlinear filtering, SIAM J. Control and Optim., 38 (2000), pp. 683-710.

[9] V.N. Kolokoltsov and V.P. Maslov, Idempotent Analysis and Its Applications, Kluwer, 1997.

[10] G.L. Litvinov, V.P. Maslov and G.B. Shpiz, Idempotent Functional Analysis: An Algebraic Approach, Mathematical Notes, 69, No. 5 (2001), pp. 696-729.

[11] W.M. McEneaney, Max-Plus Methods for Nonlinear Control and Estimation, Birkhauser, Boston, 2006.

[12] W.M. McEneaney, Distributed Dynamic Programming for DiscreteTime Stochastic Control, and Idempotent Algorithms, Preprint.

[13] W.M. McEneaney, Complexity Reduction, Cornices and Pruning, Proc. of the International Conference on Tropical and Idempotent Mathematics, G.L. Litvinov and S.N. Sergeev (Eds.), AMS, (to appear).

[14] W.M. McEneaney, A. Deshpande, S. Gaubert, Curse-of-Complexity Attenuation in the Curse-of-Dimensionality-Free Method for HJB PDEs, Proc. ACC 2008, Seattle (2008).

[15] W.M. McEneaney and P.M. Dower, A max-plus affine power method for approximation of a class of mixed $l_{\infty} / l_{2}$ value functions, Proc. 42nd IEEE Conf. on Dec. and Control, Maui (2003), pp. 2573 - 2578.

[16] W.M. McEneaney, "A Curse-of-Dimensionality-Free Numerical Method for Solution of Certain HJB PDEs", SIAM J. Control and Optim., 46 (2007), 1239-1276.

[17] W.M. McEneaney, "A Curse-of-Dimensionality-Free Numerical Method for a Class of HJB PDEs", Proc. 16th IFAC World Congress (2005).

[18] G. Collins and W.M. McEneaney, "Min-Plus Eigenvector Methods for Nonlinear $\mathrm{H}_{\infty}$ Problems with Active Control", Optimal Control, Stabilization and Nonsmooth Analysis, LNCIS, Vol. 301 Queiroz, Marcio S. de; Malisoff, Michael; Wolenski, Peter (Eds.), Springer (2004), 101-120.

[19] W.M. McEneaney, Max-Plus Eigenvector Representations for Solution of Nonlinear $H_{\infty}$ Problems: Error Analysis, SIAM J. Control and Optim., Vol. 43 (2004), 379-412.

[20] W.M. McEneaney, Max-Plus Eigenvector Representations for Solution of Nonlinear $H_{\infty}$ Problems: Basic Concepts, IEEE Trans. Auto. Control 48, (2003) pp. 1150-1163.

[21] W.M. McEneaney, Error Analysis of a Max-Plus Algorithm for a FirstOrder HJB equation, Stochastic Theory and Control, Proceedings of a Workshop held in Lawrence, Kansas, October 18-20, 2001, Lecture Notes in Control and Information Sciences, Springer-Verlag, B.PasikDuncan (Ed.) (2002), pp. 335-352.

[22] William M. McEneaney, Ali Oran and Andrew Cavender, Value-Based Tasking Controllers for Sensing Assets, Proc. AIAA Guidance, Nav. and Control Conf., Honolulu, (2008).

[23] William M. McEneaney, Ali Oran and Andrew Cavender, Value-Based Control of the Observation-Decision Process, Proc. American Control Conf., Seattle, (2008).

[24] I. Singer, Abstract Convex Analysis, Wiley-Interscience, New York, 1997.

[25] A.M. Rubinov and I. Singer, Topical and Sub-Topical Functions, Downward Sets and Abstract Convexity, Optimization 50 (2001), 307351.

[26] M. Sion, On general minimax theorems, Pacific J. Math. 8 (1958) pp. $171-176$. 